\title{
Chronic Tension Type Headache and the Impact of Myofascial Trigger Point Release in the Short Term Relief of Headache
}

\author{
Magesh Anand Doraisamy (Corresponding author) \\ Masterskill University College of Health Sciences \\ G8 Jalan kemacahaya, 11 Taman kemacahaya \\ Batu 9 Cheras, Selangor, 43200, Malaysia
}

Tel: 60-1-4924-4935Ｅ-mail: dmagesh@masterskill.edu.my; mageshanand@gmail.com

\author{
Charles prem kumar \& Anshul \\ Physiotherapy, Christian Medical College, Vellore, India
}

\author{
Chandran Gnanamuthu \\ Senior Consultant Neurologist \\ Wockhardt Hospital, 154/9, Bannerghatta Road \\ Bangalore, Karnataka, India
}

\begin{abstract}
Purpose: This study investigated the effect of myofascial release in relieving the symptoms of Chronic Tension Type Headache (CTTH) Methods: The study used a convenience sampling pretest-post test design. 31 people (7 men and 24 women, age range 18-58 years, mean age 32 years) with CTTH participated in the study. The headache sample was screened for MTrP in the cranial and pericranial muscles and was treated with myofascial release therapy. The outcomes were measured in terms of the number of headache days in a week and the headache pain level measured in visual analog scale (VAS). Analysis: The analysis was done using paired ' $\mathrm{t}$ ' test at $95 \%$ confidence interval Results: The study showed significant improvements in reduction of the number of headache days $(p<0.001)$ and the headache pain level $(p<0.001)$ following treatment. Conclusion and Implications: Myofascial release therapy to the trigger points in people with CTTH has a positive influence in decreasing pain intensity and frequency in CTTH sufferers. In future, during management of CTTH screening for myofascial trigger points and concomitant treatment using myofascial release could be considered.
\end{abstract}

Keywords: Chronic Tension Type Headache, Cranial muscles, Pericranial muscles, Myofascial trigger point, Referred pain, Myofascial pressure release

\section{Introduction}

Chronic Tension type headache $(\mathrm{CTTH})$ is one of the most prevalent forms of headache. Population-based studies suggest one year prevalence rates at $38.3 \%$ for episodic tension type headache and $2.2 \%$ for chronic tension type headache (CTTH) (Schwartz et al., 1998) Being one of the common types of primary headache affecting men and women of all ages at sometime in their lives, it often impairs an individual's productivity, social time, family dynamics etc in addition to the physical suffering.

Though there have been a number of factors associated with CTTH, muscular factors have often been considered as one of the major contributors for CTTH, scientific studies strongly suggest that there are myofascial trigger points present in the cranial \& pericranial muscles that refer pain to the head which might have a considerable role in influencing the symptoms of headache (Fernandez-de-Las-Penas et al., (2006a) ; Fernandez-de-Las-Penas et al., (2006b); Fernandez-de-Las-Penas C et al., (2007a); Fernandez-de-Las-Penas et al., (2007b); Marcus et al., 1999). A myofascial trigger point is a hyper irritable spot with in a taut band of skeletal muscle or in the fascia that is painful on compression and that can give rise to characteristic referred pain, tenderness and autonomic phenomena (Simons et al., 1999).

There are several studies relating myofascial trigger points with chronic tension type headache. The local and referred pain areas and pain characteristics evoked from temporalis muscle trigger points were assessed in people with CTTH. It was reported that the evoked local and referred pain from active trigger points (TrPs) in the 
temporalis muscle and its sensory characteristics shared similar patterns as the habitual headache pain. It was suggested that the local and referred pain from active trigger points in the temporalis muscles may constitute as one of the sources for contributing to the pain profile of CTTH. (Fernandez-de-Las-Penas et al., (2007a)).In a similar study the referred pain and pain characteristics evoked from upper trapezius muscle was investigated in 20 people with CTTH. It was reported that the evoked referred pain and its sensory characteristics shared similar patterns as their habitual headache pain consistent with active TrPs. The result suggests that the spatial summation of perceived pain and mechanical pain sensitivity exists in people with CTTH (Fernandez-de-Las-Penas et al., (2007b)).

The presence of trigger points in the suboccipital muscles were studied; it was reported that 65 percent of the people assessed showed presence of active myofascial trigger points and 35\% showed presence of latent trigger points in the suboccipital muscles. The authors also reported that CTTH subjects with active trigger points reported greater headache intensity and frequency than those with latent $\operatorname{TrPs}$ (Fernandez-de-Las-Penas et al., (2006a)). In a blinded controlled study the trigger points in upper trapezius, sternocleidomastoid and temporalis muscles were identified and divided in to active and latent trigger points. It was suggested that active TrPs in the upper trapezius, sternocleidomastoid and temporalis muscles were associated with CTTH. It was also shown that CTTH subjects with active trigger points usually reported a greater headache intensity and longer headache duration than those with latent TrPs (Fernandez - de -Las Penas et al., (2006b)).

Some characteristics of CTTH, such as pressure or band-like tightness (International Headache Society., 2004) and increased tenderness on palpation of neck and shoulder muscles resemble the descriptions of referred pain originating in trigger points (Simons et al., 1999). In the recent years studies have shown strong associations between myofascial trigger points and tension type headache. The people with TTH were shown to have a greater number of either active or latent TrPs than healthy subjects (Marcus et al., 1999). The referred pain patterns seen with active trigger points were shown to be similar to that of the type of headache in tension type headache sufferers (Fernandez-de-Las-Penas et al., 2006).

There are growing evidences to suggest that there is a strong association between tension type headache and myofascial trigger points. A detailed search in EMBASE, CINAHL, PEDro, and PUBMED from its inception until 2009 and with the search terms chronic tension type headache and myofascial trigger points revealed that until date myofascial trigger points have been associated with chronic tension type headache, predominantly in terms of diagnostic value (Fernandez-de-Las-Penas et al., (2006a); Fernandez-de-Las-Penas et al., (2006b); Fernandez-de-Las-Penas C et al., (2007a); Fernandez-de-Las-Penas et al., (2007b); Marcus et al., 1999). There are very few studies that have targeted myofascial trigger points as an entity in the management perspective of CTTH (Norman et al., 2009; Moraska and Chandler 2008). There is further need for evidence in the light of screening all the myofascial trigger points that has been shown to refer pain to the head (Simons et al., 1999) and further treat them using standard myofascial release methods and evaluate its effect in the relief of symptoms from headache.

\section{Material and methods}

\subsection{Subjects}

Thirty one people with CTTH, 7 men and 24 women, mean age 32 years, S.D 11.37, mean headache years 3.07) were recruited for the study from the outpatient consultation unit of the Department of Neurology, Christian Medical College, Vellore, India. People with CTTH were diagnosed according to the criteria of the International headache society (IHS) (International Headache Society., 2004) by an experienced neurologist. People with CTTH who had headache for at least 3 days in a week for the past four consecutive weeks were selected. The diagnosis was arrived up on the history of the patient done by interview and referring to the medical record of the subject. The exclusion criteria were, surgeries involving the cervical spine, documented Intracranial causes contributing to headache, cervical disc prolapse, and systemic illness contributing to their headache and forms other than CTTH.

All patients recruited for the study were on anti depressants with a drug dosage of $25 \mathrm{mg}$ T.amitriptilline for at least three months before the date of assessment. Due to ethical reasons the existing drug therapy with $\mathrm{T}$. amytriptiline for CTTH was not allowed to be discontinued. However the drug dosage was not increased during the assessment, treatment and during the follow up period of the study. The study was approved by the institutional authorities of Christian Medical College, Vellore. An informed consent was obtained from all the people who took part in this study. 


\subsection{Myofascial trigger point assessment}

The muscles of the head and neck that are shown to refer pain to the head (Simons et al., 1999) were selected. The muscles assessed were, sternocleidomastoid, upper trapezius, sub occipitalis, frontalis, temporalis, occipitalis and splenius capitis. The muscles were assessed by an experienced therapist well versed with the assessment and management of myofascial trigger points. The trigger point was located as per the guidelines given in the literature (Simons et al., 1999). The muscles were classified as cranial and pericranial muscles. Those muscles that have their attachments within the cranium was classified as cranial muscle and those muscles that have one of their attachment in the cranium and the other outside the cranium was classified as pericranial muscles.

The assessment and treatment for all the trigger points was performed by the same therapist following the diagnostic criteria described in the literature (Simons et al., 1999). The diagnostic criteria for a trigger point followed in this study was (1) presence of a palpable taut band in a skeletal muscle; (2) presence of a hypersensitive tender spot in the taut band; (3) reproduction of the typical referred pain pattern of the trigger point in response to compression. The above criterion has been shown to be the minimum acceptable criterions to diagnose a trigger point in the literature (Simons et al., 1999).

A trigger point was considered active if the subject had spontaneous pain at the site of the trigger point and also had spontaneous referred pain to the head in the usual site of headache (Simons et al., 1999). A trigger point was considered latent if the person did not have any spontaneous pain but had referred pain to the head in the usual site of headache up on palpation (Simons et al., 1999). If the person did not have any referred pain but tenderness in a circumscribed spot in muscle up on compression, it was considered as a tender point. Myofascial release was given to both active and latent trigger points but the tender points were not treated.

In this study the tenderness of the trigger point was assessed based on a simple tenderness scale, Grade 1 - patient complains of pain

Grade 2 - patient complains of pain and winces

Grade 3 - patient winces and withdraws the joint

Grace 4 - patient will not allow palpation of the joint.

The assessment for the trigger point was done by the same therapist throughout the study. The tenderness was elicited by applying pressure over the trigger point with the pulp of the index finger until the nail blanched. The tenderness was graded according to the response of the person. The tenderness grading was used to assess the status of the trigger point before and after treatment.

\subsection{Assessment of pain intensity and severity of headache}

The number of days of headache in a week was considered as the severity of headache and the intensity of headache was considered as the pain level during headache measured in a $10 \mathrm{~cm}$ visual analog scale. The information of number of headache days in a week was obtained by history taking through interview and from the headache records. The person with headache was asked to mark the usual headache intensity in a $10 \mathrm{~cm}$ visual analog scale. The method of recording the headache intensity and severity by the interview method has been shown to be a reliable method (Niere and Jerak 2004). All the people included in the study had a history of at least three headache days in a week.

\subsection{Treatment}

Ischemic compression (trigger point pressure release) (Simons et al., 1999) using pincer grasp or point compression, as appropriate, was performed for all the trigger points identified to produce referred pain to the cranium. Trigger point pressure release is a treatment method in which a slowly increasing, nonpainful pressure is applied over a trigger point until a barrier of tissue resistance is encountered. The contact is then maintained until the tissue barrier releases, and pressure is increased to reach a new barrier to eliminate the trigger point tension and tenderness (Simons et al., 1999). All the people with headache were treated once; all the trigger point was given one session of treatment. The person with myofascial trigger point was positioned comfortably and the muscle positioned in its neutral length. Digital pressure was applied to the trigger point for a period of approximately 90 sec.

The pressure was continued until the sensitivity of the trigger point wanes and the tension in the taut band fades. The trigger point was treated once. This was then followed by gentle passive stretches to the muscles treated for trigger points (Stretching the muscle treated for trigger point is part of the treatment protocol of myofascial trigger point treatment intervention). The muscle was stretched to its full length twice following the treatment of 
the trigger point. A home program of neck active range of motion exercises were taught to the patients and asked to practice it twice in a day for 10 repetitions during morning and evening. The exercises involve active neck flexion, neck extension, side bending to both side and rotations to either side.

\subsection{Assessment and Treatment technique (Simons et al., 1999)}

\subsubsection{Upper Trapezius}

This is a pericranial muscle with attachments from the cranium to the cervical vertebrae. Trigger points from this muscle refers pain along the postero lateral aspect of the neck, behind the ear and to the temple. For assessment and treatment the person is positioned in high sitting and the therapist can palpate the trigger point in the upper trapezius using point compression.

\subsubsection{Sterno cleido mastoid}

This is a pericranial muscle with attachments from the cranium to the sternum and the clavicle. Trigger points from the sternal division refers pain to the vertex, to the occiput, across the cheek, over the eye, to the throat and to the sternum and the clavicular division typically refers pain to the forehead and ear. For assessment and treatment, the person is positioned in high sitting; the therapist palpates the muscle using pincer compression for identifying the trigger point in the sternal fibres and Clavicular fibers.

\subsubsection{Sub Occipitals}

This is also a pericranial muscle with attachments from the cranium to the proximal cervical vertebrae. Trigger points from this muscle refers pain in to the occiput, eye and the forehead. The sub occipitals are located in the posterior neck, just below the occipital part. The muscle is attached from the occipital protuberance and occipital ridge to $\mathrm{C} 1$ and $\mathrm{C} 2$ vertebra. For assessment and treatment the patient is positioned in prone lying with the head in neutral position to mild neck extension. The therapist uses point compression to palpate for the trigger point in the sub occipitals muscle.

\subsubsection{Splenius capitis}

This is a pericranial muscle with attachments to the cranium and the cervical vertebrae. Trigger points from this muscle refer pain to the vertex of the head. For assessment and treatment the person is positioned in high sitting and the trigger point is palpated at the root of the neck using point compression.

\subsubsection{Occipitalis}

This is a cranial muscle; the attachments are entirely in the cranium. Trigger points from this muscle refers pain laterally and anterior, diffusely over the back of the head and through the cranium, causing intense pain deep in the orbit. For assessment and treatment the person is positioned in high sitting and the muscle is palpated using point compression just above the external occipital protuberance.

\subsubsection{Frontalis}

This is a cranial muscle as well; the attachments are within the cranium. Trigger points from this muscle evokes pain that spread upward and over the forehead on the same side. For assessment and treatment the person is positioned in high sitting and the muscle is palpated just above the eye brow using point compression.

\subsubsection{Temporalis}

This is a cranial muscle with the attachments in the cranium. Trigger points from this muscle refers pain throughout the temple, along the eye brow, behind the eye and can be felt in any or all of the upper teeth. For assessment and treatment, the person is positioned in high sitting and the trigger point is palpated in the temple region.

\subsection{Follow up}

The people with headache were given a headache record and asked to mark their headache profile in the record and come back after one week. The number of days they had headache in the week was taken as the severity of headache and the worst headache marked in the visual analog scale was taken as the intensity of their headache. They were asked to mark the headache intensity in a $10 \mathrm{~cm}$ visual analog scale. The number of days they had headache was taken as the severity of headache and the day in which they had the worst headache was taken as the intensity of the headache. Further the tenderness of the trigger point was assessed by the therapist and the tenderness was graded according to the tenderness grading scale to see for any relapse in the trigger point. At the time of one week follow up period no relapse of the trigger point was observed. 


\subsection{Statistical analysis}

Data were analyzed with the SPSS statistical package (11.5 versions). Paired' $t$ ' test with a $95 \%$ confidence interval was done. The severity of headache measured as the number of days of headache in a week and the intensity of headache measured as the score in the visual analog scale was compared for data obtained before and after treatment intervention. A $P$ value less than 0.05 was considered statistically significant.

\section{Results}

A total of 31 people with CTTH took part in the study, 7 men and 24 women aged between 18 to 62 years (mean 32 years, S.D 11.37, mean headache years 3.07). The mean of the headache intensity before treatment intervention was $7.28+/-1.53$ which reduced to a mean of $1.65+/-2.77$ on the $10 \mathrm{~cm}$ visual analog scale $(\mathrm{P}<$ 0.001 ). The severity (frequency) of the headache was a mean of $4.64+/-1.97$ days in a week before intervention which decreased to $0.61+/-1.58$ days in a week $(\mathrm{P}<0.001)$ after the intervention.

The patients were screened for the presence of myofascial trigger points in the sternocleidomastoid, upper trapezius, suboccipitalis, temporalis, occipitalis, frontalis, and splenius capitis. Out of the 31 people screened, 21 people with headache had myofascial trigger points in all the 3 pericranial muscles. 30 people had trigger points in the upper trapezius, 28 people had trigger point in the sternocleidomastoid and 21 had trigger points in the suboccipitals. All those who had trigger point in the suboccipitals also had trigger points in the sternocleidomastoid and upper trapezius. This is an interesting finding and needs further exploration on the relationship between these three muscles in influencing each other. 6 people had trigger points in the temporalis, and 3 each in the occipitalis and frontalis, it is also interesting to note that all those who had trigger point in the occipitalis also had trigger points in the frontalis.

\section{Discussion}

There are many factors held to be causative factors of CTTH. In this study the muscular myofascial trigger points was addressed. The trigger points might play an individual role or it could be part of the complex causes that amalgamate and cause the headache. In this study a significant reduction of headache parameters were observed upon treatment of the trigger points, which adds up to the current evidence that treatment of myofascial trigger points has a major role in the management perspective of headache.

It is imperative to understand the mechanism by which a myofascial trigger point can affect CTTH to understand the mechanism of headache relief by myofascial release. Olesen proposed that headache is due to an excess of nociceptive inputs from peripheral structures (Olesen 1991). According to his model, headache intensity is the sum of nociceptive inputs from cranial and extra cranial tissues converging on trigeminal nucleus caudalis neurons. The convergence of the nociceptive afferents from the receptive fields of cervical roots $\mathrm{C} 1-\mathrm{C} 3$ and those of the trigeminal nerve occurs in the nucleus caudalis (Piovesan et al., 2003). Continuous or prolonged nociceptive afferent input resulting in temporal and spatial summation could lead to central sensitization and pain perception in CTTH. Fernandez further updated this pain model and proposed that the myofascial trigger points are responsible for the peripheral mechanisms leading to activation or sensitization of nociceptive nerve endings by the liberation of chemical mediators (bradikinin, serotonin, substance P) (Fernandez-de-Las-penas et al., 2007; Jay et al., 2008). Hence when the trigger point is deactivated by myofascial release the afferent input from the myofascial trigger point is stopped and the headache symptoms are relieved.

Few studies have been done until date in which myofascial trigger points have been targeted in the management perspective of tension type headache. The efficacy of Botulinum toxin A was evaluated in the treatment of CTTH with myofascial trigger points producing referred head pain. Improvements were seen in headache status in the initial follow up period of 2 weeks but found a dissipation of effects by week 12 (Norman et al., 2009). This finding suggests that treatment focused at myofascial trigger points do have a role to play in management of CTTH. However this treatment method cannot be adapted by physiotherapists and the risks are relatively higher when injecting in to muscles such as suboccipitals and sternocleidomastoid in which case only a well experienced physician could do the procedure. Massage therapy in association with myofascial release of active trigger points were administered to the patients with CTTH who also had myofascial trigger points (Moraska and Chandler 2008) However in this study only the neck muscles were primarily targeted and the cranial muscles were not considered, moreover one may not want to discount the general analgesic effect of massage in the relief of headache. Hence the contribution from the myofascial trigger point component alone towards the headache would still not be clear.

In this study the treatment was focused only at the myofascial trigger points and $58 \%$ of the subjects had complete relief however $42 \%$ did not have complete relief during the follow up period. The result obtained from 
this study reflects that there could be other causes apart from myofascial trigger points such as psychological factors, osteogenic factors etc that can contribute to the symptoms of CTTH. But considering the vast extent of relief from the symptoms, it could be hypothesized that myofascial trigger points could either have a more important role than the other causes or they all form a cycle interacting and complementing each other. When one of the components is removed the other components also tends to resolve to a certain extent thus resulting in significant relief from the symptoms.

The number of people who had trigger points in the pericranial muscles was more than the cranial muscles (See table 1). In the pain model proposed by Olesen, he proposes that the CTTH is influenced by the convergence of nociceptive afferents from the receptive fields of cervical roots $\mathrm{C} 1-\mathrm{C} 3$ and also from the trigeminal nerve (Olesen 1991). The trigger points in the sternocleidomastoid, upper trapezius and suboccipitals are associated with the cervical roots rather than the trigeminal nerve. This leads to a hypothesis that the cervical roots C1-C3 might have a stronger role than the trigeminal nerve in influencing CTTH. Future studies can be targeted to delineate between the cervical and trigeminal contributions in CTTH.

The follow up period was kept quite short, primarily because the trigger point was treated in an effort to see if it had any effect at all in the headache population. Moreover the chance of relapse of the trigger points is quite high because the cause of myofascial pain syndrome was not addressed. It would have been interesting to note the changes in the headache parameters over time coupled along with mapping of the trigger points at regular intervals. It would have given a clearer picture of the association between the CTTH and trigger points.

\section{Conclusion}

Myofascial trigger points have been shown to play a significant role in CTTH. All the trigger points that referred pain to the cranium were treated and significant improvements in headache parameters were observed. A simple yet efficient method of treating myofascial trigger points was used which in many cases could be self administered too. There are some limitations in this study. First, only subjects with CTTH was selected and treated. Hence the results cannot be extrapolated to other headache disorders, such as Episodic Tension Type Headache, Chronic Daily Headache, and Migraine etc. It would be interesting to repeat the same procedure with patients suffering from other disorders in order to explore the relevance of head and neck muscle trigger points in headache. The second limitation was the small sample size and methodology. A blinded controlled trial with more samples could confirm the findings seen in this trial. However this study adds further evidence to the non pharmacologic methods for the management of Tension type headache.

\section{References}

Fernandez-de-Las-Penas, C., Alonso-Blanco, C., Cuadrado, M.L., Gerwin, R.D., Pareja, J.A. (2006). Myofascial trigger points and their relationship to headache clinical parameters in chronic tension type headache. Headache, 8 , 1264-72.

Fernandez-de-las-Penas, C., Alonso-Blanco, C., Cuadrado, M.L., Gerwin, R.D., Pareja, J.A. (2006). Myofascial trigger points in the suboccipital muscles and forward head posture in chronic tension type headache. Headache, 46, 454-460.

Fernandez-de-las-Penas, C., Alonso-Blanco, C., Cuadrado, M.L., Gerwin, R.D., Pareja, J.A. (2006). Myofascial trigger points and their relationship with headache clinical parameters in chronic tension type headache. Headache, 46, 1264-1272.

Fernández-de-las-Peñas, C., Cuadrado, M.L., Arendt-Nielsen, L., Simons, D.G., Pareja, J.A. (2007). Myofascial trigger points and sensitization: an updated pain model for tension-type headache. Cephalalgia, 27(5), 383-93.

Fernandez-de-Las-Penas, C., Ge, H.Y., Arendt-Nielson, L., Cuadrado, M.L., Pareja, J.A. (2007). The local and referred pain from myofascial trigger points in the temporalis muscle contributes to pain profile in chronic tenion type headache. Clin J Pain, 23(9), 786-92.

Fernandez-de-Las Penas, C., Ge, H.Y., Arendt-Nielsen, L., Cuadrado, M.L., Pareja, J.A. (2007). Referred pain from trapezius muscle trigger points shares similar characteristics with chronic tension type headache. Eur J Pain, $11(4), 475-82$.

IHS: Headache Classification Subcommittee of the International Headache Society. (2004). The International Classification of Headache Disorders, Cephalalgia, 24(suppl 1), 9-160.

Jay, P.S., Elizabeth, A., Gilliams, B.A. (2008). Uncovering the biochemical milieu of myofascial trigger points using in vivo microdialysis:An application of muscle pain concepts to myofascial pain syndrome. Journal of Bodywork and Movement Therapies, 12, 371-84. 
Jensen, R., Olesen, J. (1996). Initiating mechanism of experimentally induced tension-type headache. Cephalalgia, 16, 175-182.

Marcus, D.A., Scharff, L., Mercer, S., Turk, D.C. (1999). Musculoskeletal abnormalities in chronic headache: A controlled comparison of headache diagnostic groups. Headache, 39, 21-27.

Moraska, A., Chandler, C. (2008). Changes in clinical parameters with tension type headache following massage therapy: A pilot study. J Man Manip Ther, 16(2), 106-12.

Niere, K., Jerak, A. (2004). Measurement of headache frequency, intensity and duration: comparison of patient report by questionnaire and headache diary. Physiother Res Int, 9(4), 149-56.

Norman, H.R., Jerod, C., Christine, M.G., Todd, A., Smitherman., Stephan, R., Weinland., Beverley, T., Petra, J., Thomas, S.L., Timothy, T.H. (2009). Botulinum toxin A in the treatment of chronic tension-type headache with cervical myofascial trigger points: a randomized, double-blind, placebo-controlled pilot study. Headache, 49(5), $732-43$.

Olesen, J. (1991). Clinical and pathophysiological observations in migraine and tension-type headache explained by integration of vascular, supraspinal and myofascial inputs. Pain, 46, 125-132.

Piovesan, E.J., Kowacs, P.A., Oshinsky, M.L. (2003). Convergence of cervical and trigeminal sensory afferents. Curr Pain Headache Rep, 7, 377-383.

Schwartz, B.S., Stewart, W.F., Simon, D. (1998). Epidemiology of tension-type headache. JAMA, 279, 381- 383.

Simons, D.G., Travell, J., Simons, L.S. (1999). Myofascial pain and dysfunction: The trigger point manual Volume 1, Upper half of body. 2nd ed. Baltimore: Williams and Wilkins.

Table 1. Showing the number of people who had referred pain to the head from the trigger points of the muscles assessed

\begin{tabular}{|c|c|c|}
\hline Muscles & Right & Left \\
\hline Sternocleidomastoid & $\mathbf{2 8}$ & $\mathbf{2 5}$ \\
\hline Upper trapezius & $\mathbf{3 0}$ & $\mathbf{2 9}$ \\
\hline Splenius Capitis & 4 & 4 \\
\hline Suboccipitals & $\mathbf{2 1}$ & $\mathbf{2 1}$ \\
\hline Temporalis & 6 & 6 \\
\hline Occipitalis & 3 & 3 \\
\hline Frontalis & 3 & 3 \\
\hline
\end{tabular}

